\title{
RESONANT OPTICAL RECTIFICATION IN CHIRAL MOLECULAR SYSTEMS: APPLICATION TO A THREE-LEVEL MODEI.
}

\author{
S. WoźnIAK \\ Nonlinear Optics Division, Institute of Physics \\ A. Mickiewicz University, Umultowska 85, 61-614 Poznań, Poland \\ (Received February 19, 1996; in final form July 2, 1996)
}

\begin{abstract}
A molecular approach to optical rectification via the imaginary part of the second-order susceptibility related to electric dipole interaction with the radiation field is presented. A quantum-mechanical expression for the frequency dependence of the effect is obtained and its magnitude is estimated in absorption bands of a three-level model.
\end{abstract}

PACS numbers: 33.55.Fi, 42.65.An

Linear and nonlinear interactions of an electromagnetic wave with molecules of an isotropic medium induce in them multipole moments which contribute to electric polarization of the system [1]. Out of absorption bands optical rectification (OR) via the second-order susceptibility is forbidden but the fourth-order dipolar electric nonlinearity may cause OR in chiral liquids [2]. However, in absorption bands of optically active molecular system OR is allowed via the imaginary part of the second-order susceptibility related to electric dipole interaction with the radiation field [3].

We consider an isotropic molecular system in which a plane monochromatic light wave with the electric field

$$
E(z, t)=\frac{1}{2} E(\omega) \exp \left(-\mathrm{i} \omega\left(t-\frac{\tilde{n}}{c} z\right)\right)+\text { c.c. }
$$

oscillating with the circular frequency $\omega$, propagates in the $z$-direction. For a dissipative medium $\tilde{n}$ is complex

$$
\tilde{n}=n(1+\mathrm{i} / \mathrm{i})
$$

with $n$ the refractive index, and $\kappa$ - the absorption coefficient.

In this paper we are interested in the calculation of a static polarization $P(0)$ induced in the medium by nonlinear interaction of chiral molecules with the light wave. Restricting our considerations to the second-order susceptibility, we have

$$
P_{i}(0)=N \exp (-\beta z)\left\langle\tilde{a}_{i j k}(0 ; \omega,-\omega)\right\rangle E_{j}(\omega) E_{k}^{*}(\omega),
$$

where $N$ is the number of molecules per unit volume, $\tilde{\alpha}_{i j k}$ the nonlinear polarizability related to electric dipole transitions, whereas the asterisk represents the complex conjugate, the symbol \langle\rangle stands for the isotropic average [4], and

$$
\beta=\frac{2 \omega n i}{c} \text {. }
$$


In the region of absorption the tensor $\tilde{\boldsymbol{\alpha}}(0 ; \omega,-\omega)$ is complex

$$
\tilde{\alpha}_{i j k}(0 ; \omega,-\omega)=\alpha_{i j k}(0 ; \omega,-\omega)+\mathrm{i} \alpha_{i j k}^{\prime}(0 ; \omega,-\omega),
$$

where both tensors $\alpha(0 ; \omega,-\omega)$ and $\alpha^{\prime}(0 ; \omega,-\omega)$ are now real.

The polarizability tensor $\tilde{\alpha}(0 ; \omega,-\omega)$ of Eq. (3) is a time-even polar tensor of rank three and has nonzero components for molecules without a centre of symmetry. Quantum-mechanical forms of the polarizabilities $\alpha_{i j k}$ and $\alpha_{i j k}^{\prime}$ can be obtained by perturbation theory [5]. The number of nonzero and independent components depends on the specific molecular symmetry (see, for instance, Tables $4.2 \mathrm{a}$ and $4.2 \mathrm{e}$ in Ref. [6] presenting the components of a polar tensor of rank three without permutational symmetries).

The components $\tilde{\alpha}_{i j k}(0 ; \omega,-\omega)$ fulfil the additional relation

$$
\tilde{\alpha}_{i j k}(0 ; \omega,-\omega)=\tilde{\alpha}_{i k j}^{*}(0 ; \omega,-\omega)
$$

which leads to

$$
\begin{aligned}
& \alpha_{i j k}(0 ; \omega,-\omega)=\alpha_{i k j}(0 ; \omega,-\omega), \\
& \alpha_{i j k}^{\prime}(0 ; \omega,-\omega)=-\alpha_{i k j}^{\prime}(0 ; \omega,-\omega) .
\end{aligned}
$$

The relations (7) and ( 8 ) reduce the number of independent components of the polarizability tensors $\alpha_{i j k}(0 ; \omega,-\omega)$ and $\alpha_{i j k}^{\prime}(0 ; \omega,-\omega)$ for certain well-defined symmetries.

Carrying out spatial averaging of the electric dipole moments induced in the molecules of the medium we can write the $z$ component of the static polarization in the form convenient for a further discussion, namely

$$
P_{z}(0)=\frac{2 N I \sin 2 \eta \exp (-\beta z)}{3 \hbar^{2} \epsilon_{0} c} \sum_{m}^{\prime} \sum_{n>m}^{\prime} F_{m n}(\omega) \operatorname{Re}\left[\mu_{g n} \cdot\left(\mu_{n m} \times \mu_{m g}\right)\right],
$$

which is nonzero only for elliptically or circularly polarized light in absorption bands of chiral molecules, $\eta$ is the ellipticity of the light wave, $\Sigma^{\prime}$ stands for summation over all states except the ground state $|g\rangle, \mu_{m g}$, denotes an electric dipole transition between the states $|g\rangle$ and $\langle m| . F_{m n}(\omega)$ is a frequency-dependent function which has the form

$$
F_{m n}(\omega)=A_{m n}(\omega)-A_{n m}(\omega)+B_{m n}(\omega)
$$

with

$$
\begin{aligned}
& A_{m n}(\omega)=\omega\left[\Gamma_{n g}\left(\omega^{2}-\omega_{m g}^{2}\right)+\Gamma_{m g}\left(\Gamma_{m g} \Gamma_{n g}-2 \omega_{m g} \omega_{n g}\right)\right] q_{n}^{0} q_{m}, \\
& B_{m n}(\omega)=\omega\left\{2\left(\omega_{m g} \omega_{n g}+\omega^{2}+\Gamma_{m g} \Gamma_{n g}\right)\right. \\
& \times\left[\Gamma_{m g}\left(\omega_{n g}^{2}-\omega^{2}+\Gamma_{n g}^{2}\right)-\Gamma_{n g}\left(\omega_{m g}^{2}-\omega^{2}+\Gamma_{m g}^{2}\right)\right]+\left(\Gamma_{m g}-\Gamma_{n g}\right) \\
& \left.\times\left[\left(\omega_{m g}^{2}-\omega^{2}+\Gamma_{m g}^{2}\right)\left(\omega_{n g}^{2}-\omega^{2}+\Gamma_{n g}^{2}\right)+4 \omega^{2} \Gamma_{m g} \Gamma_{n g}\right]\right\} q_{m} q_{n},
\end{aligned}
$$

where

$$
\begin{aligned}
& q_{n}^{0}=\left(\omega_{n g}^{2}+\Gamma_{n g}^{2}\right)^{-1} \\
& g_{m}=\left[\left(\omega_{m g}^{2}-\omega^{2}+\Gamma_{m g}^{2}\right)^{2}+4 \omega^{2} \Gamma_{m g}^{2}\right]^{-1}
\end{aligned}
$$




$$
q_{n}=\left[\left(\omega_{n g}^{2}-\omega^{2}+\Gamma_{n g}^{2}\right)^{2}+4 \omega^{2} \Gamma_{n g}^{2}\right]^{-1},
$$

and $\Gamma_{n g}^{-1}$ is a characteristic relaxation time between the states $|n\rangle$ and $|g\rangle$.

Above, Eq. (9) is given in a form applicable for any isotropic molecular system, however, the summation over all states (except the ground state) is beset with difficulties and therefore some approximations are needed. A molecule represented by three levels is the simplest model to describe nonlinear effects related to the second-order susceptibility and this model can serve as a good approximation for molecules possessing two absorption bands in the optical region, if the remaining bands are situated far from them [7].

Now, the general equation (9) will be applied to a molecule represented by three levels ( $g$ the ground state, $a$ and $b$ the excited states). If the light frequency $\omega$ is tuned to the absorption band with the resonance frequency $\omega_{a g}$ (close to the resonance frequency) then the light-induced static polarization is given in the form

$$
\begin{aligned}
P_{z}(0) & \cong \frac{N I \sin 2 \eta \exp (-\beta z)}{3 \hbar^{2} \epsilon_{0} c} \frac{\omega \Gamma_{a g}}{\left(\Delta_{a g}^{2}+\Gamma_{a g}^{2}\right) \omega_{b g}\left(\omega_{b g}-\omega\right)} \\
& \times \operatorname{Re}\left[\mu_{g b} \cdot\left(\mu_{l a} \times \mu_{a g}\right)\right],
\end{aligned}
$$

where $\Delta_{a g}=\omega_{a y}-\omega$. A similar expression describes optical rectification in the resonance region with the frequency $\omega_{b g}$.

If the frequency $\omega$ is not close to the resonance frequency $\omega_{a g}$ or $\omega_{b g}$, the conditions $\left|\omega_{a g}-\omega\right| \gg \Gamma_{a g}$ and $\omega_{b g}-\omega \mid \gg \Gamma_{b g}$ are fulfilled, and we have

$$
\begin{gathered}
P_{z}(0)=\frac{2 N I \omega \Gamma \sin 2 \eta \exp (-\beta z)}{3 \hbar^{2} \epsilon_{0} c} \\
\times\left[\frac{\omega^{2}-\omega_{a g}^{2}-2 \omega_{a g} \omega_{b g}}{\omega_{l g}^{2}\left(\omega_{a g}^{2}-\omega^{2}\right)^{2}}-\frac{\omega^{2}-\omega_{b g}^{2}-2 \omega_{a g} \omega_{b g}}{\omega_{a g}^{2}\left(\omega_{b g}^{2}-\omega^{2}\right)^{2}}+\frac{2\left(\omega^{2}+\omega_{a g} \omega_{b g}\right)\left(\omega_{b g}^{2}-\omega_{a g}^{2}\right)}{\left(\omega_{a g}^{2}-\omega^{2}\right)^{2}\left(\omega_{b g}^{2}-\omega^{2}\right)^{2}}\right] \\
\times \operatorname{Re}\left[\mu_{g b} \cdot\left(\mu_{b a} \times \mu_{a g}\right)\right],
\end{gathered}
$$

where, for simplicity, we assumed $\Gamma_{a g}=\Gamma_{b g}=\Gamma$.

The static polarization leads to a potential difference $U$ on the plates of a capacitor introduced into the measuring cuvette along the direction of the light wave propagation

$$
U=\frac{P_{z}(0 ; z=0)-P_{z}(0 ; z=d)}{\epsilon_{0}(\epsilon-1) \beta}
$$

where $P_{z}(0 ; z=0)$ and $P_{z}(0 ; z=d)$ are the static polarizations at $z=0$ and $z=d$, respectively; $d$ is the distance between the capacitor plates (the first is situated at $z=0)$ and $\epsilon$ is the static dielectric constant.

If the light frequency $\omega$ is tuned to the resonance frequency $\omega_{a g}$ then

$$
U\left(\omega_{a g}\right) \cong \frac{N I \sin 2 \eta\left[1-\exp \left(-\beta_{a} d\right)\right] \omega_{a g}}{3 \hbar^{2} \epsilon_{0}^{2}(\epsilon-1) c \beta_{a} \Gamma_{a g} \omega_{b g}\left(\omega_{b g}-\omega_{a g}\right)} \operatorname{Re}\left[\mu_{g b} \cdot\left(\mu_{b a} \times \mu_{a g}\right)\right],
$$

where $\beta_{a}$ is the absorption parameter $\beta$ of $\mathrm{Eq}$. (4) at the frequency $\omega=\omega_{a g}$. Denoting the maximum value of the potential differences $U\left(\omega_{a g}\right)$ and $U\left(\omega_{b q}\right)$ by $U_{\max }\left(\omega_{a g}\right)$ and $U_{\max }\left(\omega_{b g}\right)$, respectively, we have the ratio

$$
R=\frac{U_{\max }\left(\omega_{a g}\right)}{U_{\max }\left(\omega_{b g}\right)} \cong \frac{\beta_{b} \Gamma_{b g} \omega_{a g}^{2}}{\beta_{a} \Gamma_{a g} \omega_{b g}^{2}},
$$

where $\beta_{b}$ stands for the absorption parameter $\beta$ at the frequency $\omega=\omega_{b g}$. 
To estimate the potential difference given by (18) together with (16) and (17) it is necessary to have available a numerical value of the parameter $\beta$ for the appropriate frequency $\omega$. We will estimate $\beta$ from the expression [8]

$$
\beta(\omega)=\frac{2 N \omega^{2}}{3 \hbar \epsilon_{0} c n} \sum_{k}^{\prime} \frac{\omega_{k g} \Gamma_{k g}\left\langle g\left|\mu_{\alpha}\right| k\right\rangle\left\langle k\left|\mu_{\alpha}\right| g\right\rangle}{\left(\omega_{k g}^{2}-\omega^{2}+\Gamma_{k g}^{2}\right)^{2}+4 \omega^{2} \Gamma_{k g}^{2}}
$$

with $\langle g|\mu| k\rangle \approx e a_{0}$, where $e$ is the electron charge and $a_{0}$ the Bohr radius.

For our estimate we consider a three-level model with resonance frequencies lying in the optical region, $\omega_{a g}=3 \times 10^{15} \mathrm{~s}^{-1}, \omega_{b g}=5 \times 10^{15} \mathrm{~s}^{-1}$, and with the damping factors $\Gamma_{a g}$ and $\Gamma_{b g}$ identical for both transitions, $\Gamma_{a g}=\Gamma_{b g}=\Gamma=$ $10^{13} \mathrm{~s}^{-1}$. Moreover, we take $N=10^{27} \mathrm{~m}^{-3}\left(10^{27}-10^{28} \mathrm{~m}^{-3}\right.$ are typical values for the number density of liquids), $n=1.5, \epsilon=10$ and the light intensity $I=$ $10^{11} \mathrm{~W} \mathrm{~m}^{-2}$. For a distance $d$ between the capacitor plates fulfilling the relation $d \gg \beta^{-1}$ we get the following values of the potential difference $U$ at different frequencies $\omega: \omega=10^{15} \mathrm{~s}^{-1} \rightarrow U=3 \mu \mathrm{V} ; \omega=2.5 \times 10^{15} \mathrm{~s}^{-1} \rightarrow U=30 \mu \mathrm{V}$; $\omega=3 \times 10^{15} \mathrm{~s}^{-1} \rightarrow U=60 \mu \mathrm{V} ; \omega=4 \times 10^{15} \mathrm{~s}^{-1} \rightarrow U=175 \mu \mathrm{V} ; \omega=5 \times$ $10^{15} \mathrm{~s}^{-1} \rightarrow U=100 \mu \mathrm{V} ;$ and $\omega=7 \times 10^{15} \mathrm{~s}^{-1} \rightarrow U=15 \mu \mathrm{V}$.

The potential difference $U$ given by Eq. (18) depends, in general, on the number density $N$. Ilowever, it is independent of $N$ if $d \gg \beta^{-1}$, because the absorption parameter $\beta$ (Eq. (21)) and the polarization $P_{z}(0 ; z=0)$ are proportional to $N$.

The optical rectification effect can be detected if the frequency $\omega$ of the optical beam is tuned over the absorption band of chiral molecules. The above results show that the maximum magnitude of the potential difference $U$ appears not at a resonance frequency where the absorption parameter $\beta$ is high (e.g., $\beta=1.4 \times 10^{7} \mathrm{~m}^{-1}$ at $\omega=\omega_{l g}$ in the three-level model discussed) but rather out

of resonance (in our case at a frequency $\omega$ lying between $\omega_{a g}$ and $\omega_{b g}$ ), where the parameter $\beta$ is smaller (e.g., in the above model $\beta=2.3 \times 10^{3} \mathrm{~m}^{-1}$ at $\left.\omega=4 \times 10^{15} \mathrm{~s}^{-1}\right)$.

\section{References}

[1] S. Kielich, Proc. Phys. Soc. 86, 709 (1965).

[2] S. Woźniak, G. Wagnière, Optics Commun. 114, 131 (1995).

[3] N.I. Koroteev, JETP 79, 681 (1994).

[1] S. Kielich, Acta Phys. Pol. 31, 929 (1967).

[5] B.J. Orr, J.F. Ward, Mol. Phys. 20, 513 (1971).

[6] L.D. Barron, Molecular Light Scaltering and Optical Activity, Cambridge University Press, Cambridge 1982.

[7] K.E. Süsse, S. Wożniak, G. Wagnière, Optics Commun. 100, 374 (1993).

[8] S. Wó́niak, R. Zawodny, Acta Phys. Pol. A 61, 175 (1982). 